\title{
Associação de sintomas depressivos e ansiosos com gravidade da migrânea
}

\author{
Association of depressive and anxiety symptoms with migraine severity \\ Paula de Siqueira Ramos', João Gabriel Gamez Borges da Costa', Rian Avelino Mancini', \\ Rodrigo Santiago Gomez², Antonio Lúcio Teixeira ${ }^{1,2 *}$, Izabela Guimarães Barbosa ${ }^{2 *}$
}

\section{RESUMO}

Objetivo: O objetivo do presente estudo foi avaliar a associação do sono, depressão e ansiedade em pacientes com migrânea. Métodos: Cinquenta pacientes do sexo feminino, provenientes de um centro terciário de tratamento de cefaleias, com o diagnóstico de migrânea segundo os critérios da International Headache Society, foram incluídas neste estudo. As pacientes foram avaliadas com os seguintes instrumentos: Migraine Disability Assessment test (MIDAS), Headache Impact Test (HIT), Hospital Anxiety (HADS-A) and Depression Scale (HAD-D), Índice de Qualidade do Sono de Pittsburgh (IQSP) e Escala de Sonolência de Epworth (ESE). Resultados: As pacientes apresentaram alta prevalência de sintomas de ansiedade (60\%) e de depressão (42\%), má qualidade do sono (80\%) e sonolência diurna (36\%). Foi demonstrada correlação positiva entre a gravidade dos sintomas de ansiedade e HIT ( $p=0,018 ; \rho=0,334)$, ESE $(p=0,002 ; \rho=0,426)$ e IQSP $(p=0,002 ; \rho=0,426)$. Correlação positiva também foi demonstrada entre a gravidade dos sintomas depressivos e HIT ( $p<0,001 ; \rho=0,532)$, ESE $(p=$ $0,035 ; \rho=0,299)$ e IQSP ( $p=0,016, \rho=0,34)$. Não houve associação entre a qualidade do sono,

\section{Palavras-chave}

Migrânea, depressão, ansiedade, distúrbios do sono. a sonolência diurna e a gravidade da migrânea. A pontuação na HAD-D foi o principal preditor de impacto grave da migrânea. Conclusão: Apesar da alta frequência de distúrbios do sono, o principal fator relacionado ao impacto da migrânea foi a gravidade dos sintomas depressivos.

\section{ABSTRACT}

Objective: The aim of the present study was to evaluate the association of sleep, depression and anxiety symptoms in migraine patients. Methods: Fifty female patients diagnosed with migraine according to the International Headache Society criteria were recruited from a headache referral clinic. Patients were evaluated using the following instruments: Migraine Disability Assessment test (MIDAS), Headache Impact Test (HIT), Hospital Anxiety (HADS-A) and Depression scale (HAD-D), Pittsburgh Sleep Quality Index (PSQI) and Epworth Sleepiness Scale (ESS). Results: Patients presented higher prevalence of anxiety (60\%) and depressive (42\%) symptoms, poor quality of sleep (80\%) and daytime sleepiness (36\%). A positive correlation was shown between anxiety symptoms and HIT ( $p=0.018 ; \rho=0.334)$, ESS ( $p=0.002$; $\rho=0.426)$, and PSQI $(p=0.002 ; \rho=0.426)$. A positive correlation was also shown between

1 Universidade Federal de Minas Gerais (UFMG), Faculdade de Medicina.

2 UFMG, Faculdade de Medicina, Laboratório Interdisciplinar de Investigação Médica, Divisão de Neurociências.

Recebido em

28/10/2014

Aprovado em

$13 / 4 / 2015$
* Estes autores contribuíram igualmente na realização deste artigo.

Endereço para correspondência: Izabela Guimarães Barbosa

Faculdade de Medicina, sala 281,

Laboratório Interdisciplinar de Investigação Médica

Av. Alfredo Balena, 190

30130-100 - Belo Horizonte, MG, Brasil

E-mail: izabelagb@gmail.com 


\section{Keywords}

Migraine, depression, anxiety, sleep disorders. depressive symptoms and HIT ( $p<0.001 ; \rho=0,532)$, ESS $(p=0.035 ; \rho=0.299)$ and PSQI $(p=0.016, \rho=0.34)$. There was no association between quality of sleep, daytime sleepiness, and migraine severity. HAD-D score was the only predictor associated with substantial or severe migraine impact. Conclusion: Despite the elevated frequency of sleep disorders, the main factor associated with migraine impact was the severity of depressive symptoms.

\section{INTRODUÇÃO}

Entre 50\% e 75\% dos adultos com idade entre 18 e 65 anos têm pelos menos um tipo de dor de cabeça no intervalo de um ano, e a migrânea, popularmente conhecida como enxaqueca, é causa de cefaleia em mais de $10 \%$ dos pacientes' Migrânea é a forma mais comum de cefaleia primária nos serviços médicos, sendo responsável por expressivo impacto na qualidade de vida e na redução da capacidade laborativa das pessoas acometidas ${ }^{2,3}$.

Entre os possíveis fatores precipitantes das crises de migrânea, incluem-se consumo de bebidas alcoólicas e determinados alimentos, menstruação, fadiga e alterações do sono. Privação do sono e hipersonia são apontadas como desencadeadoras de crises de migrânea em mais de 50\% dos pacientes ${ }^{4-7}$. Além disso, as alterações do sono são queixas frequentes em pacientes com migrânea, e pelo menos 50\% dos pacientes relatam queixas persistentes de insônia inicial e intermediária ${ }^{8}$. Curiosamente, além de desencadear crises de migrânea, o sono pode ser utilizado como estratégia abortiva da crise de cefaleia nesses pacientes ${ }^{8,9}$. Há, portanto, uma relação bidirecional entre migrânea e sono.

Os mecanismos neurobiológicos dessa relação não são bem conhecidos. Estudos com tomografia por emissão de pósitrons (PET) demonstraram hiperativação do hipotálamo, centro do controle do ritmo circadiano, durante a crise de migrânea, sugerindo que o hipotálamo seria importante desencadeador de múltiplos estados de dor ${ }^{10,11}$. Outra hipótese que poderia justificar a íntima relação entre a migrânea e o sono envolve a identificação de disfunção serotoninérgica em ambas as condições. Estudos da década de 1960 revelaram altos níveis do ácido 5-hidroxi-indolacético, um metabólito da serotonina, na urina de pacientes com migrânea ${ }^{12,13}$. Durante as crises de migrânea, elevadas concentrações séricas de serotonina também foram descritas ${ }^{14}$. Fármacos que atuam nos receptores de serotonina, como os triptanos, são eficazes no tratamento da migrânea ${ }^{15}$. Além disso, expressão diferencial de receptores de serotonina (5-HT) 1A, 5-HT1B, 5-HT2A tem sido relacionada a fases distintas do ciclo sono-vigília' ${ }^{16}$.

Pacientes com migrânea apresentam elevada incidência de transtornos psiquiátricos. Entre os transtornos psiquiátricos com maior prevalência, estão os transtornos ansiosos (57\%), o transtorno depressivo (31\%) e a distimia (21\%) ${ }^{17}$. Parece haver associação bidirecional entre migrânea e transtornos ansiosos e depressivos mediada por mecanismos di- versos, como alterações em monoaminas, disfunção imune e fatores genéticos compartilhados. Ademais, alterações de sono estão entre os critérios diagnósticos de ansiedade e depressão do DSM-5 e CID-10. Caracteristicamente, os quadros depressivos cursam com insônia terminal ou despertares precoces, e pacientes com transtornos de ansiedade cursam com insônia inicial e/ou insônia de manutenção do sono ${ }^{18,19}$.

São poucos os estudos que investigaram simultaneamente a possível associação entre migrânea, sono e transtornos ansiosos e depressivos ${ }^{20}$. Portanto, o presente estudo tem como objetivo avaliar a influência do sono e dos transtornos ansiosos e depressivos, de forma independente, na gravidade da migrânea.

\section{MÉTODOS}

Foi realizado um estudo transversal com 50 pacientes do sexo feminino com o diagnóstico de migrânea, segundo os critérios da International Headache Society (IHS 2004) ${ }^{21}$, atendidas consecutivamente no Ambulatório de Cefaleias do Serviço de Neurologia do Hospital das Clínicas da UFMG (AmbCefHC/UFMG). Foram excluídas pacientes com cefaleia tensional, cefaleias secundárias, epilepsia, gestantes e puérperas.

As pacientes foram entrevistadas utilizando-se questionário estruturado para a coleta de dados demográficos (idade, sexo, escolaridade, tipo de trabalho), clínicos gerais (diabetes mellitus, hipertensão arterial sistêmica e dislipidemia) e sobre as características clínicas da cefaleia, incluindo tempo de doença, presença de aura e abuso de analgésicos. Todas as pacientes tiveram seu peso $(\mathrm{kg})$ e altura $(\mathrm{cm})$ mensurados no momento da entrevista, sendo calculado o índice de massa corporal (IMC) de cada uma delas por meio da fórmula $\left(\mathrm{kg} / \mathrm{cm}^{2}\right)$.

Foram utilizados os seguintes instrumentos para avaliar o impacto da cefaleia: (i) Headache Impact Test (HIT) ${ }^{22}$, que avalia o impacto da dor de cabeça sobre a vida do paciente; a escala é pontuada de 36 a 78, sendo dividida em quatro grupos: (1) pouco ou nenhum impacto ( $\leq 49$ pontos), (2) algum impacto (entre 50 e 55 pontos), (3) impacto moderado (entre 56 e 59 pontos) e (4) impacto grave ( $\geq 60$ pontos); (ii) Migraine Disability Assesment test (MIDAS) ${ }^{23}$, para avaliar o período e a frequência de cada crise e a incapacidade causada por ela. Os pacientes são categorizados em quatro grupos: (1) incapacidade mínima ( $\leq 5$ pontos), (2) incapacidade leve 
(entre 6 e 10 pontos); (3) incapacidade moderada (entre 11 e 20 pontos) e (4) incapacidade grave ( $\geq 21$ pontos).

A qualidade do sono foi avaliada pelo Índice de Qualidade do Sono de Pittsburgh (IQSP), que consiste na avaliação da qualidade do sono durante o último mês e é pontuado de 0 a 21. As pacientes foram divididas entre dois grupos: boa qualidade do sono ( $\leq 5$ pontos) e má qualidade do sono $(<5 \text { pontos })^{23-25}$. A escala de sonolência de Epworth foi utilizada para investigar a presença de sonolência diurna, que é definida por e um valor maior ou igual a 11 pontos ${ }^{26}$.

Para avaliar a presença de sintomas de ansiedade e depressão, foi utilizada a escala Hospital Anxiety and Depression Scale $(\mathrm{HADS})^{27}$. Para identificação dos sintomas ansiosos, foi utilizada a subescala HAD-ansiedade (HAD-A) e para os sintomas depressivos, a subescala HAD-depressão (HAD-D). Cada uma das subescalas contém sete questões com pontuações que variam de 0 a 21 pontos. Caso a pontuação seja igual ou maior que 8 , o paciente pode ser classificado como apresentando provável ansiedade (HAD-A $\geq 8$ ) e/ou provável depressão (HAD-D $\geq 8$ ).

As análises estatísticas foram realizadas com o software SPSS versão 17.0 (SPSS Inc., Chicago, IL, EUA). Estatística descritiva foi utilizada para apresentar as características sociodemográficas e clínicas da amostra. Associação entre variáveis dicotômicas foi avaliada com o Teste do Qui-Quadrado de Pearson ou o teste exato de Fisher, quando apropriado. Todas as variáveis foram testadas quanto à normalidade de distribuição por meio do teste de Shapiro-Wilk, e todos os dados apresentaram distribuição não normal. Portanto, as diferenças entre os dois grupos foram comparadas com o teste de Mann-Whitney. Realizou-se correlação de Spearman entre frequência e impacto da migrânea com qualidade de sono, sonolência diurna, sintomas de ansiedade e depressão. Todos os valores de p foram bicaudais e o nível de significância de $a=0,05$ foi escolhido.

As diferenças entre as pacientes categorizadas em dois grupos - sem impacto ou com impacto baixo (HIT $\geq 56$ pontos); e com impacto moderado ou grave (HIT $\geq 56$ pontos) foram examinadas por meio de regressão logística backward. De acordo com o procedimento de eliminação backward, as variáveis com maior valor de p foram progressivamente excluídas do modelo. O modelo final contém variáveis com nível de significância $\leq 0,05$. A qualidade do ajuste do modelo final foi testada pelo método de Hosmer-Lemeshow, e o odds ratio e o intervalo de confiança de 95\% são mostrados para cada variável independente no modelo.

\section{RESULTADOS}

\section{Dados sociodemográficos e clínicos da população estudada}

Os dados sociodemográficos e clínicos encontram-se na tabela 1. A média de idade das pacientes foi de 41,52 (DP $\pm 13,74)$. As pacientes apresentaram tempo de evolução médio da doença de 19,68 anos (DP $\pm 14,09$ ). A maioria das pacientes $(59,10 \%)$ apresentava o diagnóstico de migrânea sem aura. Em relação à atividade laboral, 78\% das pacientes estavam empregadas; dessas, 92\% trabalhavam no período diurno. Entre as pacientes, 56\% apresentavam comorbidades: hipertensão arterial sistêmica (18\%), diabetes mellitus (8\%), dislipidemia (14\%), sobrepeso (32\%) e obesidade (18\%).

Tabela 1. Dados demográficos, clínicos e específicos de migrânea da população de pacientes com migrânea

\begin{tabular}{|c|c|}
\hline & Pacientes com migrânea $(n=50)$ \\
\hline Idade em anos (média \pm DP) & $41,52 \pm 13,74$ \\
\hline Tempo de evolução da doença em anos (média \pm DP) & $19,68 \pm 14,09$ \\
\hline Migrânea sem aura (frequência \%) & 59,10 \\
\hline HIT (média \pm DP) & $59,10 \pm 8,22$ \\
\hline MIDAS (média \pm DP) & $34,20 \pm 56,88$ \\
\hline Comorbidades (frequência \%) & 56,00 \\
\hline Hipertensão arterial sistêmica (frequência \%) & 18,00 \\
\hline Dislipdemia (frequência \%) & 14,00 \\
\hline Diabetes mellitus (frequência \%) & 8,00 \\
\hline Sobrepeso (frequência \%) & 32,00 \\
\hline Obesidade (frequência \%) & 18,00 \\
\hline
\end{tabular}

DP: desvio-padrão; HIT: Headache Impact Test; MIDAS: Migraine Disability Impact test.

Cerca de metade das pacientes apresentou, em seu histórico, algum episódio de abuso de analgésicos. Nenhuma paciente fazia abuso atual de analgésicos ou de outras substâncias, e 74\% das pacientes faziam uso episódico de analgésicos. Quanto ao uso de fármacos profiláticos: 44\% estavam em uso de antidepressivos tricíclicos, 34\%, de anticonvulsivantes e 30\%, de betabloqueadores. Em relação a outros psicofármacos, 20\% das pacientes estavam em uso de hipnóticos-sedativos e 18\%, de inibidores seletivos da recaptura de serotonina.

A média de pontos obtidos no MIDAS, referentes à incapacidade da migrânea, foi de 34,20 (DP \pm 56,88). Quando categorizadas em relação à incapacidade, $42 \%$ das pacientes apresentavam incapacidade grave, 20\%, incapacidade moderada, 8\%, incapacidade leve e 30\%, incapacidade mínima. Em relação ao HIT, a média encontrada foi de 59,10 (DP \pm $8,22)$ e, quando categorizadas no que se refere ao impacto, $48 \%$ apresentaram impacto grave, $12 \%$, impacto moderado, $32 \%$, algum impacto e $8 \%$, pouco ou nenhum impacto.

Os dados referentes aos sintomas de qualidade do sono, presença de sonolência diurna excessiva e sintomas de ansiedade e depressão encontram-se na tabela 2. Quanto à investigação de sonolência diurna excessiva, a média obtida foi de 7,76 (DP $\pm 5,42$ ). Em relação à qualidade do sono, $80 \%$ das pacientes apresentavam sono ruim e apenas 20\% tinham sono bom (média \pm DP, 7,96 $\pm 4,75$ ). Entre as pacientes 
entrevistadas, 10\% procuraram assistência médica, pelo menos uma vez durante a vida, por causa de problemas relacionados ao sono.

Tabela 2. Dados sobre sintomas de ansiedade e depressão, qualidade do sono e sonolência diurna

\begin{tabular}{lc}
\hline & Pacientes com migrânea $(\mathrm{n}=50)$ \\
\hline HAD-A (média \pm DP) & $9,62( \pm 4,78)$ \\
HAD-D (média \pm DP) & $7,60( \pm 5,83)$ \\
Epworth (média \pm DP) & $7,76 \pm 5,42$ \\
Sonolência diurna & \\
Sim (frequência \%) & 36 \\
Não (frequência \%) & 64 \\
Pittisburgh (média \pm DP) & $7,96 \pm 4,75$ \\
Qualidade do sono & \\
Boa (frequência \%) & 20 \\
Má (frequência \%) & 80 \\
\hline
\end{tabular}

HAD-A: Hospital Anxiety Scale; HAD-D: Hospital Depression Scale.

Em relação ao HAD-A, a média de pontuação foi de 9,62 $( \pm 4,78)$, e $60 \%$ das pacientes apresentavam provável quadro de ansiedade. Em relação à HAD-D, a média de pontuação foi de 7,60 ( $\pm 5,83$ ), e 42\% das pacientes apresentavam provável quadro depressivo.

Sintomas ansiosos correlacionaram-se positivamente com sonolência diurna ( $p=0,012, \rho=0,352)$, qualidade do sono $(p=0,002 ; \rho=0,426)$, sintomas depressivos $(p<0,001 ; \rho=$ $0,706)$ e impacto relacionado à migrânea ( $p=0,018 ; \rho=0,334)$. Sintomas depressivos correlacionaram-se positivamente com qualidade de sono $(p=0,016, \rho=0,34)$, sonolência diurna ( $p=$ $0,035 ; \rho=0,299)$ e impacto relacionado à migrânea $(p<0,001$; $\rho=0,532)$. Há positiva correlação entre o número de dias de cefaleia por mês e o impacto da cefaleia $(p<0,001 ; \rho=0,495)$ e a incapacidade gerada pela cefaleia ( $p<0,001 ; \rho=0,557)$. Não houve associação entre sonolência diurna ou qualidade de sono e número de dias de cefaleia por mês.

\section{Comparação de pacientes com migrânea sem impacto e com impacto}

Os pacientes foram divididos em dois grupos, sendo categorizados como: pacientes com baixo ou nenhum impacto $(\mathrm{HIT}<$ 55 pontos) e pacientes com impacto moderado ou grave (HIT $\geq 56$ pontos). Dezenove pacientes (38\%) apresentavam baixo ou nenhum impacto e 31 (62\%) apresentavam impacto moderado ou grave. Não houve diferença entre os grupos em relação à idade, à presença de comorbidades, ao IMC, ao uso de fármacos ou à presença de sonolência diurna (Tabela 3).

Pacientes do grupo com impacto moderado ou grave da migrânea apresentaram pontuações mais elevadas nas subescalas de ansiedade (HAD-A) $(p=0,03)$ e de depressão (HAD-D) ( $p<0,001)$ quando comparadas ao grupo de pacientes com baixo ou nenhum impacto.

\section{Modelo de regressão}

Um modelo de regressão logística foi realizado para avaliar a probabilidade de apresentar impacto moderado ou grave de migrânea. O modelo inicial apresentou três variáveis independentes (diabetes mellitus, HAD-A e HAD-D). O modelo final foi estatisticamente significativo $-X^{2}(3, N=50)=16,98$, $p=0,001$ - indicando que foi capaz de distinguir o grupo de pacientes com baixo ou nenhum impacto do grupo de pacientes com impacto moderado ou grave. $O$ modelo explicou entre 37,9\% (Cox-Snell R-square) e 51,5\% (Nagelkerke R-square) da variação. Como mostrado na tabela 5, apenas a gravidade dos sintomas depressivos fez uma contribuição significativa para o modelo.

Tabela 3. Dados demográficos e clínicos nos grupos de baixo ou nenhum impacto e de impacto substancial ou grave da migrânea

\begin{tabular}{|c|c|c|c|}
\hline & Baixo ou nenhum impacto $(n=19)$ & Impacto substancial ou grave $(n=31)$ & Valorp \\
\hline Idade em anos (média \pm DP) & $44,94 \pm 14,79$ & $39,41 \pm 12,85$ & $0,16^{\dagger}$ \\
\hline Tempo de doença (média \pm DP) & $18,47 \pm 14,80$ & $20,41 \pm 13,83$ & $0,64^{\dagger}$ \\
\hline Migrânea sem aura (frequência \%) & 58,82 & 40,74 & $1,00^{t+t}$ \\
\hline $\begin{array}{l}\text { Abuso de analgésicos, pelo menos um episódio no passado } \\
\text { (frequência \%) }\end{array}$ & 47,36 & 54,83 & $0,77^{+t+}$ \\
\hline Comorbidades (frequência \%) & 68,42 & 48,38 & $0,24^{\dagger++}$ \\
\hline Hipertensão arterial sistêmica (frequência \%) & 15,78 & 19,35 & $1,00^{t+t+}$ \\
\hline Diabetes mellitus (frequência \%) & 21,05 & 0 & $0,17^{t+t}$ \\
\hline Dislipidemia (frequência \%) & 21,05 & 9,61 & $0,40^{t+t+}$ \\
\hline IMC categorizado & & & $0,83^{++}$ \\
\hline Normal (frequência \%) & 47,36 & 51,61 & \\
\hline Sobrepeso (frequência \%) & 36,84 & 29,03 & \\
\hline Obesidade (frequência \%) & 15,78 & 19,35 & \\
\hline
\end{tabular}

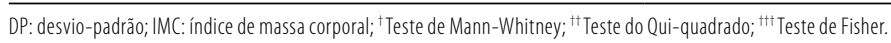


Tabela 4. Dados da gravidade da migrânea, da qualidade do sono e da sonolência diurna nos grupos de baixo ou nenhum impacto e de impacto substancial ou grave da migrânea

\begin{tabular}{lccc}
\hline & $\begin{array}{c}\text { Baixo ou nenhum } \\
\text { impacto }(\mathbf{n}=19)\end{array}$ & $\begin{array}{c}\text { Impacto substancial } \\
\text { ou grave }(\mathbf{n}=31)\end{array}$ & Valor p \\
\hline MIDAS (média \pm DP) & $9,21 \pm 11,79$ & $49,51 \pm 67,55$ & $<0,001 \dagger$ \\
HAD-A (média \pm DP) & $7,47 \pm 4,27$ & $10,93 \pm 4,66$ & $0,03 \dagger$ \\
HAD-A (frequência \%) & 47,37 & 67,74 & $0,24 \dagger$ \\
HAD-D (média \pm DP) & $4,10 \pm 3,46$ & $9,74 \pm 5,98$ & $<0,001 \dagger$ \\
HAD-D (frequência \%) & 15,79 & 58,06 & $0,004 \dagger$ \\
Epworth & $7 \pm 6,04$ & $8,22 \pm 5,05$ & $0,26 \dagger$ \\
$\begin{array}{l}\text { Sonolência excessiva } \\
\text { Epworth }\end{array}$ & 21,05 & 45,16 & $0,085 \dagger$ \\
$\begin{array}{l}\text { Pittisburgh } \\
\text { Qualidade do sono }\end{array}$ & $6,78 \pm 4,11$ & $8,67 \pm 5,03$ & $0,30 \dagger$ \\
$\begin{array}{l}\text { Qualidade do sono boa } \\
\text { Má qualidade do sono }\end{array}$ & 15,78 & 22,58 & $0,72 \dagger \dagger$ \\
$\begin{array}{l}\text { Já procurou assistência } \\
\text { médica devido a } \\
\text { problemas do sono? }\end{array}$ & 54,21 & 77,41 & \\
\hline
\end{tabular}

MIDAS: Migraine Disability Impact Test; DP: desvio-padrão; HAD-A: Hospital Anxiety Scale; HAD-D: Hospital Depression Scale; ${ }^{\dagger}$ Teste de Mann-Whitney; ${ }^{\dagger \dagger}$ Teste de Fisher.

Tabela 5. Modelo de regressão logística predizendo impacto substancial ou grave um pacientes com migrânea.

\begin{tabular}{|c|c|c|c|c|c|c|c|c|}
\hline & \multirow[t]{2}{*}{ B } & \multirow[t]{2}{*}{ S.E. } & \multirow[t]{2}{*}{ Wald } & \multirow[t]{2}{*}{ Df } & \multirow{2}{*}{$\begin{array}{l}\text { Valor } \\
\text { dep }\end{array}$} & \multirow{2}{*}{$\begin{array}{l}\text { Odds } \\
\text { Ratio }\end{array}$} & \multicolumn{2}{|c|}{$\begin{array}{c}\text { Intervalo de } \\
\text { confiança de 95\% }\end{array}$} \\
\hline & & & & & & & Baixo & Elevado \\
\hline HAD-D & 0,276 & 0,088 & 9,21 & 1 & 0,002 & 1,318 & 1,109 & 1,567 \\
\hline Constante & $-1,321$ & 0,62 & 4,53 & 1 & 0,033 & 0,267 & & \\
\hline
\end{tabular}

\section{DISCUSSÃO}

O presente estudo avaliou a influência da presença da qualidade do sono e de sintomas de ansiedade e de depressão na gravidade da migrânea em uma amostra de pacientes de um serviço terciário. Foram identificadas elevadas frequências de má qualidade de sono e de sonolência diurna, assim como de sintomas ansiosos e depressivos. O principal achado de nosso estudo foi a destacada influência dos sintomas depressivos sobre o impacto relacionado à migrânea.

Em relação às características clínicas da amostra estudada, elas assemelharam-se às descritas em outras pesquisas realizadas em cenários assistenciais quanto à frequência de migrânea sem aura ${ }^{28-30}$ e à incapacidade relacionada à migrânea ${ }^{24,28}$. Por outro lado, a presente amostra apresentou maior tempo de evolução e maior gravidade da migrânea (avaliada pelo MIDAS) quando comparada a de outros estudos ${ }^{28,31}$. A longa evolução e a maior gravidade da migrânea podem ser justificadas pelo fato de a amostra ser exclusivamente feminina, considerando que outros estudos incluíram participantes do sexo feminino e do sexo masculino. Ressalta-se que a migrânea é mais frequente, mais grave e tem maior risco de transição do tipo episódico para o crônico em pacientes do sexo feminino quando comparados aos pacientes do sexo masculino $29,32,33$.

Estudos têm indicado que a prevalência de má qualidade do sono em pacientes com migrânea encontra-se entre $60 \%$ e $85 \%$ 12,25,30,34, resultado semelhante ao encontrado neste estudo. Em relação à sonolência diurna, a prevalência em torno de $35 \%$ das pacientes está de acordo com o descrito na literatura ${ }^{30,34}$. Pacientes com migrânea também possuem maior número de despertares noturnos, insônia inicial e maior latência do estágio 1 do sono, quando comparados a pacientes sem migrânea ${ }^{25}$. Alguns estudos demonstraram correlação positiva entre a gravidade da migrânea e a qualidade do sono, o que não foi observado no presente estudo ${ }^{28,35}$. Embora não seja evidente a explicação para essa discordância, possivelmente características clínicas próprias das populações estudadas podem explicá-la em parte.

Diversos estudos populacionais e em centros terciários têm evidenciado associação entre migrânea e transtornos psiquiátricos ${ }^{36,37}$. Observamos que os sintomas ansiosos e depressivos se correlacionaram com o impacto da migrânea, o que vem sendo descrito por outros estudos ${ }^{38,39}$. Na análise multivariada, evidencia-se que os sintomas depressivos são o principal fator determinante do impacto da migrânea. Com efeito, migrânea e depressão se relacionam intimamente. Pacientes com migrânea têm duas a quatro vezes mais chance de apresentar depressão ${ }^{37}$. A depressão é apontada como fator preditor de cronificação da migrânea, influenciando sobremaneira seu prognóstico ${ }^{38,40}$. Assim, pacientes com migrânea devem ser ativamente investigados quanto à presença de sintomas e episódios depressivos e, quando detectados, adequadamente tratados. Antonaci et al. ${ }^{41}$ sugerem três possíveis relações entre migrânea e sintomas depressivos e ansiosos: (i) sintomas depressivos/ansiosos podem ser fator causal para o desenvolvimento de migrânea, principalmente para migrânea crônica; (ii) migrânea pode ser o fator causal para o desenvolvimento de sintomas depressivos/ansiosos, em particular a presença de dor crônica pode facilitar o desenvolvimento desses sintomas; (iii) podem existir fatores etiológicos comuns subjacentes à ocorrência das condições, como alterações de sistemas de neurotransmissores e de circuitos cerebrais ${ }^{41,42}$.

Podem ser considerados limitações do trabalho: o pequeno tamanho amostral; a ausência de grupo controle; a aferição dos sintomas de ansiedade e depressão por meio de inventários em detrimento do diagnóstico clínico psiquiátrico. Além disso, o estudo foi realizado em um ambulatório de serviço terciário, com uma amostra exclusivamente do sexo feminino. Por se tratar de um estudo transversal, não é 
possível estabelecer uma relação de causalidade entre sintomas depressivos/ansiosos e o impacto da migrânea. Um desenho de estudo longitudinal seria mais adequado para o estudo de causalidade. Por outro lado, este é o primeiro estudo brasileiro que investiga simultaneamente a influência dos distúrbios do sono, da ansiedade e da depressão na gravidade da migrânea. Foi realizada também cuidadosa avaliação clínica das pacientes, incluindo comorbidades que são geralmente negligenciadas.

\section{CONCLUSÃO}

Como conclusão, observa-se que, embora a má qualidade de sono e a sonolência diurna sejam comuns entre os pacientes, a gravidade dos sintomas depressivos é possivelmente o principal fator relacionado ao impacto da migrânea.

\section{CONTRIBUIÇÕES INDIVIDUAIS}

Paula de Siqueira Ramos, Antonio Lúcio Teixeira e Izabela Guimarães Barbosa - Desenharam o estudo, realizaram as análises estatísticas e escreveram o esboço do artigo.

João Gabriel Gamez Borges da Costa, Rian Avelino Mancini e Rodrigo Santiago Gomez - Recrutaram os sujeitos para o estudo.

Todos os autores contribuíram e aprovaram o manuscrito final.

\section{AGRADECIMENTOS}

Este trabalho foi financiado pelo Conselho Nacional de Desenvolvimento Científico e Tecnológico (CNPq, Brasil), Fundação de Amparo à Pesquisa do Estado de Minas Gerais (Fapemig, Brasil) e Coordenação de Aperfeiçoamento de Pessoal de Nível Superior (Capes, Brasil).

\section{CONFLITOS DE INTERESSE}

Não há

\section{REFERÊNCIAS}

1. World Health Organization Media Centre. Fact Sheet no 277, October 2012. Disponível em: <http://www.who.int/mediacentre/factsheets/fs277/en/>. Acesso em: 24 jun. 2014.

2. Silva Jr. AA, Tavares RM, Lara RP, Faleiros BE, Vasconcelos LPB, Gómez RS, et al. Frequência das cefaleias primárias na comunidade e em centros de cuidados especializados. Rev Dor. 2013;14(2):84-7.

3. Lipton RB, Hamelsky SW, Kolodner KB, Steiner TJ, Stewart WF. Migraine, quality of life, and depression: a population-based case-control study. Neurology. 2000;55(5):629-35.
4. lerusalimschy R, Moreira Filho PF. Fatores desencadeantes de crises de migrânea em pacientes com migrânea sem aura. Arq Neuropsiquiatr. 2002;60(3-A):609-13.

5. Yagihara F, Lucchesi LM, Smith AKA, Speciali JG. Primary headaches and their relationship with sleep. Sleep Sci. 2012 5(1):28-32.

6. Bittencourt LRA, Silva RS, Santos RF, Pires MLN, Mello MT. Sonolência excessiva. Rev Bras Psiquiatr. 2005;271(Supl I):16-21.

7. Mollaoğlu M. Trigger factors in migraine patients. J Health Psychol. 2013;18(7):984-94.

8. Kelman MDL, Rains JC. Headache and sleep: examination of sleep patterns and complaints in a large clinical sample of migraineurs. Headache. 2005;45(7):904-10.

9. WHO technical meeting on sleep and health. WHO Regional Office for Europe. European Centre for Environment and Health Bonn Office. Bonn, Germany, 22-24 January 2004. Disponível em: <http://www.euro.who.int/__data/assets/pdf_file/0008/114101/ E84683.pdf>. Acesso em: 24 jun. 2014.

10. Holland PR. Headache and sleep: shared pathophysiological mechanisms. Cephalalgia. 2014;34(10):725-44

11. Denuelle M, Fabre N, Payoux P, Chollet F, Geraud G. Hypothalamic activation in spontaneous migraine attacks. Headache. 2007;47(10):1418-26.

12. Vincent MB. Fisiopatologia da enxaqueca. Arq Neuropsiquiatr. 1998;56(4):841-51.

13. Ferrari MD, Saxena PR. On serotonin and migraine: a clinical and pharmacological review. Cephalalgia. 1993;13(3):151-65.

14. Kalaycıoğlu E, Gökdeniz T, Aykan AC, Gürsoy MO, Gül I, Ayhan N, et al. Evaluation of right ventricle functions and serotonin levels during headache attacks in migraine patients with aura. Int J Cardiovasc Imaging. 2014;30(7):1255-63.

15. Dussor G. Serotonin, 5 HT1 agonists, and migraine: new data, but old questions still not answered. Curr Opin Support Palliat Care. 2014;8(2):137-42.

16. Hornung JP. The human raphe nuclei and the serotonergic system. J Chem Neuroanat. 2003;26(4):331-43.

17. Teixeira AL, Costa EA, da Silva AA Jr, dos Santos IA, Gómez RS, Kummer A, et al. Psychiatric comorbidities of chronic migraine in community and tertiary care clinic samples. J Headache Pain. 2012;13(7):551-5.

18. Lucchesi LM, Pradella-Hallinan M, Lucchesi M, Moraes WAS. 0 sono em transtornos psiquiátricos. Rev Bras Psiquiatr. 2005;27(Supl I):27-32.

19. Chellappa SL, Araújo JF. O sono e os transtornos do sono na depressão. Rev Psiq Clín. 2007;34(6);285-9.

20. Sancisi E, Cevoli S, Vignatelli L, Nicodemo M, Pierangeli G, Zanigni S, et al. Increased prevalence of sleep disorders in chronic headache: a case-control study. Headache. 2010;50(9):1464-72.

21. Headache Classification Subcommittee of the International Headache Society. The International Classification of Headache Disorders: 2nd edition. Cephalalgia. 2004;24(Suppl 1):9-160.

22. Bayliss MS, Dewey JE, Dunlap I, Batenhorst AS, Cady R, Diamond ML, et al. A study of the feasibility of Internet administration of a computerized health survey: the headache impact test (HIT). Qual Life Res. 2003;12(8):953-61.

23. Stewart WF, Lipton RB, Dowson AJ, Sawyer J. Development and testing of the Migraine Disability Assessment (MIDAS) Questionnaire to assess headache-related disability. Neurology. 2001;56(6 Suppl 1):S20-8.

24. Buysse DJ, Reynolds (F 3rd, Monk TH, Berman SR, Kupfer DJ. The Pittsburgh Sleep Quality Index: a new instrument for psychiatric practice and research. J Psychiatr Res. 1989;28(2):193-213.

25. Walters B, Hamer JD, Smitherman TA. Sleep disturbance and affective comorbidity among episodic migraineurs. Headache. 2014;54(1):116-24.

26. Karthik N, Sinha S, Taly AB, Kulkarni GB, Ramachandraiah CT, Rao S. Alteration in polysomnographic profile in 'migraine without aura' compared to healthy controls. Sleep Med. 2013;14(2):211-4.

27. Bertolazzi AN, Fagondes SC, Hoff LA, Pedro VD, Barreto SSM, Johns MW. Validação da escala de sonolência de Epworth em português para uso no Brasil. J Bras Pneumol. 2009;35(9):877-83. 
28. Botega NJ, Bio MR, Zomignani MA, Garcia JRC, Pereira WAB. Transtornos do humor em enfermaria de clínica médica e validação de escala de medida (HAD) de ansiedade e depressão. Rev Saúde Pública. 1995;29(5):355-63.

29. Yalinay Dikmen P, Yavuz BG, Aydinlar El. The relationship between migraine, depression, anxiety, stress and sleep disturbances. Acta Neurol Belg. 2014 [in press].

30. Sevillano-García MD, Manso-Calderón R, Cacabelos-Pérez P. Comorbidity in the migraine: depression, anxiety, stress and insomnia. Rev Neurol. 2007;45(7):400-5.

31. SeidelS, Hart IT,Weber M,MattereyS, Paul A, RiedererF,etal.;PAMINA Study Group. Quality of sleep, fatigue and daytime sleepiness in migraine - a controlled study. Cephalalgia. 2009;29(6):662-9.

32. Smitherman TA, McDermott MJ, Buchanan EM. Negative impact of episodic migraine on a university population: quality of life, functional impairment, and comorbid psychiatric. Headache. 2011;51(4):581-9.

33. Finocchi C, Strada L. Sex-related differences in migraine. Neurol Sci. 2014;35(Suppl 1):207-13.

34. Stuginski-Barbosa J, Dach F, Bigal M, Speciali JG. Chronic pain and depression in the quality of life of women with migraine - a controlled study. Headache. 2012;52(3):400-8.

35. Barbanti P, Aurilia C, Egeo G, Fofi L, Vanacore N. A case-control study on excessive daytime sleepiness in chronic migraine. Sleep Med. 2013;14(3):278-81.
36. Sadeghniiat K, Rajabzadeh A, Ghajarzadeh M, Ghafarpour M. Sleep quality and depression among patients with migraine. Acta Med Iran. 2013;51(11):784-8.

37. Jette N, Patten S, Williams J, Becker W, Wiebe S. Comorbidity of migraine and psychiatric disorders: a national population-based study. Headache. 2008;48(4):501-16.

38. Pompili M, Di Cosimo D, Innamorati M, Lester D, Tatarelli R, Martelletti P. Psychiatric comorbidity in patients with chronic daily headache and migraine: a selective overview including personality traits and suicide risk. J Headache Pain. 2009;10(4):283-90.

39. Buse D, Manack A, Serrano D, Reed M, Varon S, Turkel C, et al. Headache impact of chronic and episodic migraine: results from the American Migraine Prevalence and Prevention study. Headache. 2012;52(1):3-17.

40. Ashina S, Serrano D, Lipton RB, Maizels M, Manack AN, Turkel CC,et al. Depression and risk of transformation of episodic to chronic migraine. J Headache Pain. 2012;13(8):615-24.

41. Antonaci F, Nappi G, Galli F, Manzoni GC, Calabresi P, Costa A. Migraine and psychiatric comorbidity: a review of clinical findings. J Headache Pain. 2011;12(2):115-25.

42. Gudmundsson LS, Scher Al, Sigurdsson S, Geerlings MI, Vidal JS, Eiriksdottir G, et al. Migraine, depression, and brain volume: the AGES-Reykjavik Study. Neurology. 2013;80(23):2138-44 